Pacific

Journal of

Mathematics

MULTIPLICITY OF SOLUTIONS FOR A CLASS OF RESONANT $p$-LAPLACIAN DIRICHLET PROBLEMS

Evgenia H. Papageorgiou and Nicolaos S. Papageorgiou 


\title{
MULTIPLICITY OF SOLUTIONS FOR A CLASS OF RESONANT $p$-LAPLACIAN DIRICHLET PROBLEMS
}

\author{
Evgenia H. Papageorgiou and Nicolaos S. Papageorgiou
}

\begin{abstract}
We consider nonlinear Dirichlet problems driven by the $p$-Laplacian, which are resonant at $+\infty$ with respect to the principal eigenvalue. Using a variational approach based on the critical point theory, we show that the problem has three nontrivial smooth solutions, two of which have constant sign (one positive, the other negative). In the semilinear case, assuming stronger regularity on the nonlinear perturbation $f(z, \cdot)$ and using Morse theory, we show that the problem has at least four nontrivial smooth solutions, two of constant sign.
\end{abstract}

\section{Introduction}

Let $Z \subseteq \mathbb{R}^{N}$ be a bounded domain with a $C^{2}$-boundary $\partial Z$. In this paper, we study the following nonlinear Dirichlet problem driven by the $p$-Laplacian differential operator:

$$
\begin{aligned}
-\Delta_{p} x(z) & =f(z, x(z)) \quad \text { a.e. on } Z, \\
\left.x\right|_{\partial Z} & =0 .
\end{aligned}
$$

Here, $\Delta_{p}$ denotes the $p$-Laplace differential operator, namely,

$$
\Delta_{p} u=\operatorname{div}\left(\|D u\|^{p-2} D u\right), \quad 1<p<\infty .
$$

The aim of this work is to prove the existence of three nontrivial smooth solutions when resonance occurs at infinity and the Euler functional of the problem need not be coercive. Recently, three-solution theorems for the Dirichlet $p$-Laplacian were proved in [Carl and Perera 2002; Liu 2006; Liu and Liu 2005; Papageorgiou and Papageorgiou 2007; Zhang et al. 2004]. In all these works, either nonresonance is assumed or the Euler functional is coercive or both. In this work, in addition to the resonance condition at $+\infty$ with respect to the principal eigenvalue $\lambda_{1}>0$ of $\left(-\Delta_{p}, W_{0}^{1, p}(Z)\right)$ near the origin, we require that the quotient (slope) $\frac{F(z, x)}{|x|^{p}}$ (here $F(z, x)=\int_{0}^{x} f(z, s) d s$ the primitive of $\left.f(z, \cdot)\right)$ stays strictly above $\lambda_{2}>0$,

MSC2000: 35J65, 58E05.

Keywords: $p$-Laplacian, resonant problem, mountain pass theorem, second deformation theorem, Morse theory. 
the second eigenvalue of $\left(-\Delta_{p}, W_{0}^{1, p}(Z)\right)$. This way, we can take advantage of an alternative variational characterization of $\lambda_{2}>0$ by Cuesta, Figueiredo and Gossez [Cuesta et al. 1999]. Our approach is variational and based on the critical point theory. In the special case $p=2$ (semilinear problem), using in addition Morse theory and stronger regularity conditions on $f(z, \cdot)$, we are able to produce four nontrivial smooth solutions.

\section{Mathematical background}

We start by recalling some elements from critical point theory and from Morse theory, which we will need in the sequel. So, let $X$ be a Banach space and let $X^{*}$ be its topological dual. By $\langle\cdot, \cdot\rangle$ we denote the duality brackets for the pair $\left(X^{*}, X\right)$. Given $\varphi \in C^{1}(X)$, we say that $\varphi$ satisfies the Palais-Smale condition (the $P S$-condition for short) if every $\left\{x_{n}\right\}_{n \geq 1} \subseteq X$ such that $\left\{\varphi\left(x_{n}\right)\right\}_{n \geq 1}$ is bounded and $\varphi^{\prime}\left(x_{n}\right) \rightarrow 0$ in $X^{*}$ as $n \rightarrow \infty$ admits a strongly convergent subsequence.

The following minimax principle is known in the literature as the mountain pass theorem.

Theorem 2.1. If $\varphi \in C^{1}(X), \varphi$ satisfies the PS-condition, $x_{0}, x_{1} \in X$,

$$
\left\|x_{1}-x_{0}\right\|>r>0, \quad \max \left\{\varphi\left(x_{0}\right), \varphi\left(x_{1}\right)\right\}<\inf \left\{\varphi(x):\left\|x-x_{0}\right\|=r\right\}=c_{r}
$$

and

$$
c=\inf _{\gamma \in \Gamma} \max _{0 \leq t \leq 1} \varphi(\gamma(t))
$$

where $\Gamma=\left\{\gamma \in C([0,1], X): \gamma(0)=x_{0}, \gamma(1)=x_{1}\right\}$, then $c \geq c_{r}$ and $c$ is a critical value of $\varphi$, that is, there exists $x \in X$ such that $\varphi(x)=c$ and $\varphi^{\prime}(x)=0$.

Another result from critical point theory, which we will use in the analysis of problem (1-1), is the so-called second deformation theorem [Chang 1993, p. 23]. Let $\varphi \in C^{1}(X)$ and $c \in \mathbb{R}$. We define

$$
\begin{aligned}
\varphi^{c} & =\{x \in X: \varphi(x) \leq c\} \quad \text { as the sublevel set of } \varphi \text { at } c \\
K & =\left\{x \in X: \varphi^{\prime}(x)=0\right\} \quad \text { as the critical set of } \varphi \text { and } \\
K_{c} & =\{x \in K: \varphi(x)=c\} \quad \text { as the critical set of } \varphi \text { at the level } c .
\end{aligned}
$$

In the next theorem (the second deformation theorem) we allow $b=+\infty$ in which case $\varphi^{b} \backslash K_{b}=X$.

Theorem 2.2. If $\varphi \in C^{1}(X), \varphi$ satisfies the PS-condition, $a \in \mathbb{R}, a<b \leq+\infty$, $\varphi$ has no critical values in $(a, b)$ and $\varphi^{-1}(a)$ contains at most a finite number of critical points of $\varphi$, then there exists a deformation

$$
h:[0,1] \times\left(\varphi^{b} \backslash K_{b}\right) \rightarrow \varphi^{b}
$$

such that 
(a) $h\left(1, \varphi^{b} \backslash K_{b}\right) \subseteq \varphi^{a}$,

(b) $h(t, x)=x$ for all $(t, x) \in[0,1] \times \varphi^{a}$ and

(c) $\varphi(h(t, x)) \leq \varphi(h(s, x))$ for all $s, t \in[0,1], s \leq t$ and all $x \in \varphi^{b} \backslash K_{b}$.

Let $\left(Y_{1}, Y_{2}\right)$ be a topological pair with $Y_{2} \subseteq Y_{1} \subseteq X$. For every integer $k \geq 0$, by $H_{k}\left(Y_{2}, Y_{1}\right)$ we denote the $k$-th relative singular homology group of $\left(Y_{1}, Y_{2}\right)$ with coefficients in $\mathbb{Z}$. The critical groups of $\varphi \in C^{1}(X)$ at an isolated critical point $x_{0} \in X$ with $\varphi\left(x_{0}\right)=c$ are defined by

$$
C_{k}\left(\varphi, x_{0}\right)=H_{k}\left(\varphi^{c} \cap U, \varphi^{c} \cap U \backslash\left\{x_{0}\right\}\right) \quad \text { for all } k \geq 0,
$$

where $U$ is a neighborhood of $x_{0}$ such that

$$
K \cap \varphi^{c} \cap U=\left\{x_{0}\right\}
$$

(see [Chang 1993; Mawhin and Willem 1989]). The excision property of singular homology implies that this definition of critical groups is independent of the particular neighborhood $U$ we use.

Suppose that $\varphi \in C^{1}(X)$ satisfies the PS-condition and $-\infty<\inf \varphi(K)$. Choosing $c<\inf \varphi(K)$, we define the critical groups of $\varphi$ at infinity by

$$
C_{k}(\varphi, \infty)=H_{k}\left(X, \varphi^{c}\right) \quad \text { for all } k \geq 0
$$

(see [Bartsch and Li 1997]). If $K$ is finite, we set

$$
\begin{aligned}
M(t, x) & =\sum_{k \geq 0} \operatorname{rank} C_{k}(\varphi, x) t^{k} \quad \text { for all } x \in K, \\
P(t, \infty) & =\sum_{k \geq 0} \operatorname{rank} C_{k}(\varphi, \infty) t^{k} .
\end{aligned}
$$

Then the Morse relation holds

$$
\sum_{x \in K} M(t, x)=P(t, \infty)+(1+t) Q(t)
$$

where

$$
Q(t)=\sum_{k \geq 0} \beta_{k} t^{k}
$$

is a formal series with nonnegative integer coefficients (see [Chang 1993, p. 36] and [Mawhin and Willem 1989, p. 184]).

Let $X=H$ be a Hilbert space, $x \in H$ is a critical point of $\varphi \in C^{1}(H)$ and for $U$ a neighborhood of $x, \varphi \in C^{2}(U)$. Then the Morse index of $\varphi$ is defined as the supremum of the dimensions of the vector subspaces of $H$ on which $\varphi^{\prime \prime}(x)$ is negative definite. 
Definition 2.3. A map $A: X \rightarrow X^{*}$ is said to be of type $(S)_{+}$if for any sequence $\left\{x_{n}\right\}_{n \geq 1} \subseteq X$ for which $x_{n} \stackrel{w}{\rightarrow} x$ in $X$ and

$$
\limsup _{n \rightarrow \infty}\left\langle A\left(x_{n}\right), x_{n}-x\right\rangle \leq 0
$$

one has $x_{n} \rightarrow x$ in $X$.

Let $A: W_{0}^{1, p}(Z) \rightarrow W^{-1, p^{\prime}}(Z)=W_{0}^{1, p}(Z)^{*}\left(\frac{1}{p}+\frac{1}{p^{\prime}}=1\right)$ be the nonlinear map corresponding to $-\Delta_{p}$, namely,

$$
\langle A(x), y\rangle=\int_{Z}\|D x\|^{p-2}(D x, D y)_{\mathbb{R}^{N}} d z \quad \text { for all } x, y \in W_{0}^{1, p}(Z) .
$$

Hereafter by $\langle\cdot, \cdot\rangle$ we denote the duality brackets for the pair

$$
\left(W^{-1, p^{\prime}}(Z), W_{0}^{1, p}(Z)\right) \text {. }
$$

For the map A, we have the following result (see [Gasiński and Papageorgiou 2006] for example).

Proposition 2.4. The map $A: W_{0}^{1, p}(Z) \rightarrow W^{-1, p^{\prime}}(Z)$ defined by (2-2) is maximal monotone, strictly monotone and of type $(S)_{+}$.

Finally let us recall some basic facts about the spectrum of negative Dirichlet $p$ Laplacian denoted by $\left(-\Delta_{p}, W_{0}^{1, p}(Z)\right)$. For details and additional references, see [Cuesta 2001; Lê 2006]. Of course, if $p=2$ then $\Delta_{p}=\Delta$, the usual Laplace differential operator defined on $H_{0}^{1}(Z)$. We consider the following nonlinear eigenvalue problem:

$$
\begin{aligned}
-\Delta_{p} u(z) & =\lambda|u(z)|^{p-2} u(z) \quad \text { a.e. on } Z, \\
\left.u\right|_{\partial Z} & =0 .
\end{aligned}
$$

Every $\lambda \in \mathbb{R}$ for which problem (2-3) has a nontrivial solution is said to be an eigenvalue of $\left(-\Delta_{p}, W_{0}^{1, p}(Z)\right)$. The smallest eigenvalue $\lambda_{1}$ of $\left(-\Delta_{p}, W_{0}^{1, p}(Z)\right)$ is positive, simple and admits the following variational characterization

$$
\lambda_{1}=\inf \left\{\frac{\|D u\|_{p}^{p}}{\|u\|_{p}^{p}}: u \in W_{0}^{1, p}(Z), u \neq 0\right\} .
$$

Let $u_{1}$ be the $L^{p}$-normalized eigenfunction corresponding to $\lambda_{1}>0$. We know that $u_{1} \in C_{0}^{1}(\bar{Z})$ (see [Lieberman 1988]). The Banach space $C_{0}^{1}(\bar{Z})$ is an ordered Banach space with the order cone

$$
C_{+}=\left\{u \in C_{0}^{1}(\bar{Z}): u(z) \geq 0 \text { for all } z \in \bar{Z}\right\} .
$$

This cone has a nonempty interior given by

$$
\operatorname{int} C_{+}=\left\{u \in C_{0}^{1}(\bar{Z}): u(z)>0 \text { for all } z \in Z, \frac{\partial u}{\partial n}(z)<0 \text { on } \partial Z\right\},
$$


where $n$ denotes the outward unit normal on $\partial Z$. The nonlinear strong maximum principle of Vázquez [1984] implies that $u_{1} \in \operatorname{int} C_{+}$.

The Ljusternik-Schnirelmann theory in addition to $\lambda_{1}>0$ provides a whole strictly increasing sequence $\left\{\lambda_{k}\right\}_{k \geq 1} \subseteq \mathbb{R}_{+}$of eigenvalues for problem (2-3), known as the LS-eigenvalues of $\left(-\Delta_{p}, W_{0}^{1, p}(Z)\right)$. If $p=2$ (linear eigenvalue problem) then the LS-eigenvalues are all the eigenvalues of $\left(-\Delta, H_{0}^{1}(Z)\right)$. If $p \neq 2$ then we do not know if this is true. Nevertheless we know that $\lambda_{2}$ is the second eigenvalue of $\left(-\Delta_{p}, W_{0}^{1, p}(Z)\right)$. So the Ljusternik-Schnirelmann theory provides a variational characterization of $\lambda_{2}>0$. However, for our purposes, that characterization is not convenient. Instead, we will use an alternative one produced by Cuesta, Figueiredo and Gossez [Cuesta et al. 1999]. More precisely, let

$$
\begin{aligned}
\partial B_{1}^{L^{p}} & =\left\{u \in L^{p}(Z):\|u\|_{p}=1\right\}, \\
S & =W_{0}^{1, p}(Z) \cap \partial B_{1}^{L^{p}}
\end{aligned}
$$

endowed with the relative $W_{0}^{1, p}(Z)$-topology and

$$
\hat{\Gamma}=\left\{\hat{\gamma} \in C([-1,1], S): \hat{\gamma}(-1)=-u_{1}, \hat{\gamma}(1)=u_{1}\right\} .
$$

Then

$$
\lambda_{2}=\inf _{\hat{\gamma} \in \hat{\Gamma}} \max _{-1 \leq t \leq 1}\|D \hat{\gamma}(t)\|_{p}^{p}
$$

Finally, if $p=2$ and $m \in L^{\infty}(Z), m^{+} \neq 0$ (weight function), we consider the linear eigenvalue problem

$$
-\Delta u(z)=\lambda m(z) u(z) \quad \text { a.e. on } Z,\left.\quad u\right|_{\partial Z}=0 .
$$

This problem has a sequence $\left\{\hat{\lambda}_{k}(m)\right\}_{k \geq 1}$ of positive eigenvalues

$$
\hat{\lambda}_{1}(m)<\hat{\lambda}_{2}(m)<\cdots<\hat{\lambda}_{k}(m) \rightarrow+\infty
$$

and a sequence $\left\{\hat{\lambda}_{-k}(m)\right\}_{k \geq 1}$ of negative eigenvalues

$$
0>\hat{\lambda}_{-1}(m)>\hat{\lambda}_{-2}(m)>\cdots>\hat{\lambda}_{-k}(m) \rightarrow-\infty .
$$

We know that $\hat{\lambda}_{1}(m)$ is simple, isolated and

$$
\hat{\lambda}_{1}(m)=\inf \left\{\frac{\|D u\|_{2}^{2}}{\int_{Z} m u^{2} d z}: u \in H_{0}^{1}(Z), u \neq 0\right\} .
$$

Similarly for $\hat{\lambda}_{-1}(m)$. If $m \equiv 1$, then $\hat{\lambda}_{k}(m)=\lambda_{k}$ for $k \in \mathbb{Z} \backslash\{0\}$. 


\section{The nonlinear problem}

In this section, using a variational approach, we produce three nontrivial smooth solutions, two of which have constant sign.

The nonlinearity hypotheses on $f(z, x)$ are the following:

$\underline{H_{1}}: f: Z \times \mathbb{R} \rightarrow \mathbb{R}$ is a function such that $f(z, 0)=0$ a.e. on $Z$.

(i) For all $x \in \mathbb{R}, z \rightarrow f(z, x)$ is measurable.

(ii) For almost all $z \in Z, x \rightarrow f(z, x)$ is continuous.

(iii) For almost all $z \in Z$ and all $x \in \mathbb{R}$,

$$
|f(z, x)| \leq a(z)+c|x|^{r-1}
$$

with $a \in L^{\infty}(Z)_{+}, c>0$ and

$$
p<r<p^{*}= \begin{cases}\frac{N p}{N-p} & \text { if } N>p, \\ +\infty & \text { if } p \geq N .\end{cases}
$$

(iv) For almost all $z \in Z$ and all $x \geq 0, f(z, x) \geq 0$ and if $F(z, x)=\int_{0}^{x} f(z, s) d s$ then

$$
\lim _{x \rightarrow+\infty} \frac{p F(z, x)}{|x|^{p}}=\lambda_{1} \quad \text { and } \quad \lim _{x \rightarrow+\infty}\left(p F(z, x)-\lambda_{1} x^{p}\right)=-\infty,
$$

both uniformly for almost all $z \in Z$.

(v) There exist $a<0$ and $\xi>0$ such that for almost all $z \in Z, f(z, a)=0$, $f(z, x) \leq 0$ for all $x \in[a, 0]$ and

$$
x \rightarrow \xi|x|^{p-2} x+f(z, x)
$$

is nondecreasing on $[a, 0]$.

(vi) There exist $\delta_{0}>0$ and $\xi_{0}>\lambda_{2}$ such that

$$
F(z, x) \geq \frac{\xi_{0}}{p}|x|^{p} \quad \text { for a.a. } z \in Z, \text { all }|x| \leq \delta_{0} .
$$

Remark 3.1. Hypothesis $H_{1}$ (iv) implies that at $+\infty$ we have resonance with respect to $\lambda_{1}>0$ from the left. The hypotheses on $f(z, \cdot)$ on the negative semiaxis are minimal and allow the Euler functional to be strongly indefinite in the negative direction. Specifically, we only assume $H_{1}(v)$. Nevertheless, this condition with suitable truncation techniques and with the use of the nonlinear maximum principle of Vázquez [1984] leads to a negative solution of (1-1). We point out that the conditions on the two semiaxes are asymmetric. 
Example 3.2. The following function $f(x)$ satisfies hypotheses $H_{1}$ (for the sake of simplicity we drop the $z$-dependence):

$$
f(x)= \begin{cases}\hat{\xi}|x|^{p-2} x-\hat{\xi}|x|^{q-2} x & \text { if } x \leq 0, \\ \hat{\xi}|x|^{p-2} x & \text { if } x \in[0,1], \\ \lambda_{1}|x|^{p-2} x+c|x|^{\tau-2} x & \text { if } x \geq 1,\end{cases}
$$

with $\tau<p<q<p^{*}, \hat{\xi}>\lambda_{2}$ and $c=\hat{\xi}-\lambda_{1}$.

First using truncation and variational techniques, we will produce two nontrivial smooth solutions of constant sign (one positive and the other negative). To this end, we introduce the following truncation of the nonlinearity $f(z, \cdot)$ :

$$
f_{+}(z, x)= \begin{cases}0 & \text { if } x \leq 0 \\ f(z, x) & \text { if } x \geq 0 .\end{cases}
$$

We set

$$
F_{+}(z, x)=\int_{0}^{x} f_{+}(z, s) d s
$$

and introduce the functional $\varphi_{+}: W_{0}^{1, p}(Z) \rightarrow \mathbb{R}$ defined by

$$
\varphi_{+}(x)=\frac{1}{p}\|D x\|_{p}^{p}-\int_{Z} F_{+}(z, x(z)) d z \quad \text { for all } x \in W_{0}^{1, p}(Z) .
$$

Evidently $\varphi_{+} \in C^{1}\left(W_{0}^{1, p}(Z)\right)$.

Proposition 3.3. If hypotheses $H_{1}$ hold then $\varphi_{+}$is coercive.

Proof. We proceed by contradiction. So suppose that $\varphi_{+}$is not coercive. Then we can find $\left\{x_{n}\right\}_{n \geq 1} \subseteq W_{0}^{1, p}(Z)$ such that

(3-1) $\left\|x_{n}\right\| \rightarrow \infty$ as $n \rightarrow \infty$ and $\varphi_{+}\left(x_{n}\right) \leq M_{1}$ for some $M_{1}>0$, all $n \geq 1$.

Let $y_{n}=x_{n} /\left\|x_{n}\right\|$, for $n \geq 1$. Then $\left\|y_{n}\right\|=1$ for all $n \geq 1$ and we may assume that

$$
\begin{aligned}
y_{n} \stackrel{w}{\rightarrow} y & \text { in } W_{0}^{1, p}(Z) \quad \text { and } \quad y_{n} \rightarrow y \text { in } L^{p}(Z) \text { as } n \rightarrow \infty \\
& \Rightarrow y_{n}^{ \pm} \stackrel{w}{\rightarrow} y^{ \pm} \text {in } W_{0}^{1, p}(Z) \text { and } y_{n}^{ \pm} \rightarrow y^{ \pm} \text {in } L^{p}(Z) \text { as } n \rightarrow \infty .
\end{aligned}
$$

By virtue of hypotheses $H_{1}$ (iii)-(iv), we have

$$
F_{+}(z, x) \leq \frac{\lambda_{1}}{p}\left(x^{+}\right)^{p}+c_{1} \quad \text { for a.a. } z \in Z \text {, all } x \in \mathbb{R} \text { and some } c_{1}>0 .
$$

From (3-1) we have

$$
\begin{aligned}
M_{1} & \geq \frac{1}{p}\left\|D x_{n}^{+}\right\|_{p}^{p}+\frac{1}{p}\left\|D x_{n}^{-}\right\|_{p}^{p}-\int_{Z} F_{+}\left(z, x_{n}(z)\right) d z \\
& \geq \frac{1}{p}\left\|D x_{n}^{+}\right\|_{p}^{p}+\frac{1}{p}\left\|D x_{n}^{-}\right\|_{p}^{p}-\frac{\lambda_{1}}{p}\left\|x_{n}^{+}\right\|_{p}^{p}-c_{2}
\end{aligned}
$$


for some $c_{2}>0$, all $n \geq 1$ (see (3-3))

$$
\begin{aligned}
& \Rightarrow M_{1} \geq \frac{1}{p}\left\|D x_{n}^{-}\right\|_{p}^{p}-c_{2} \text { for all } n \geq 1(\text { see }(2-4)) \\
& \Rightarrow\left\{x_{n}^{-}\right\}_{n \geq 1} \subseteq W_{0}^{1, p}(Z) \text { is bounded. }
\end{aligned}
$$

Therefore $\left\|x_{n}^{+}\right\| \rightarrow \infty, y^{-}=0$,

$$
y_{n}^{+} \stackrel{w}{\rightarrow} y \text { in } W_{0}^{1, p}(Z) \quad \text { and } \quad y_{n}^{+} \rightarrow y \text { in } L^{p}(Z), y \geq 0 \text { (see (3-2)). }
$$

From (3-4) and (3-5) we have

$$
\frac{1}{p}\left\|D x_{n}^{+}\right\|_{p}^{p}-\frac{\lambda_{1}}{p}\left\|x_{n}^{+}\right\|_{p}^{p} \leq M_{2}
$$

for some $M_{2}>0$, all $n \geq 1$

$$
\Rightarrow \frac{1}{p}\left\|D y_{n}^{+}\right\|_{p}^{p}-\frac{\lambda_{1}}{p}\left\|y_{n}^{+}\right\|_{p}^{p} \leq \frac{M_{2}}{\left\|x_{n}^{+}\right\|^{p}} \quad \text { for all } n \geq 1 .
$$

Passing to the limit as $n \rightarrow \infty$ and using (3-6), we obtain

$$
\|D y\|_{p}^{p} \leq \lambda_{1}\|y\|_{p}^{p} \Rightarrow y=0 \text { or } y=\eta u_{1} \text { for some } \eta>0 \text { (recall } y \geq 0 \text { ). }
$$

If $y=0$ then from (3-6) and (3-7) it is clear that

$$
\left\|D y_{n}^{+}\right\|_{p} \rightarrow 0 \Rightarrow y_{n}^{+} \rightarrow 0 \text { in } W_{0}^{1, p}(Z) \Rightarrow y_{n} \rightarrow 0 \text { in } W_{0}^{1, p}(Z) \quad \text { (see (3-5)), }
$$

a contradiction to the fact that $\left\|y_{n}\right\|=1$ for all $n \geq 1$.

If $y=\eta u_{1}$ then recalling that $u_{1}(z)>0$ for all $z \in Z$ we have $x_{n}^{+}(z) \rightarrow+\infty$ for almost all $z \in Z$ and so, by virtue of $H_{1}$ (iv), we have

$$
F_{+}\left(z, x_{n}^{+}(z)\right)-\frac{1}{p} \lambda_{1} x_{n}^{+}(z)^{p} \rightarrow-\infty \text { for a.a. } z \in Z \text {, as } n \rightarrow \infty .
$$

From (3-4) and (3-5), we have

$$
\frac{1}{p}\left\|D x_{n}^{+}\right\|_{p}^{p}-\frac{\lambda_{1}}{p}\left\|x_{n}^{+}\right\|_{p}^{p}-\int_{Z}\left(F_{+}\left(z, x_{n}^{+}(z)\right)-\frac{1}{p} \lambda_{1} x_{n}^{+}(z)^{p}\right) d z \leq M_{3}
$$

for some $M_{3}>0$, all $n \geq 1$

$$
\Rightarrow-\int_{Z}\left(F_{+}\left(z, x_{n}^{+}(z)\right)-\frac{\lambda_{1}}{p} x_{n}^{+}(z)^{p}\right) d z \leq M_{3} \quad \text { (see (2-4)). }
$$

If in (3-9) we pass to the limit as $n \rightarrow \infty$ and use Fatou's lemma and (3-8), we reach a contradiction. This proves that $\varphi_{+}$is coercive.

Also we consider the following truncation of $f(z, \cdot)$ :

$$
\hat{f}(z, x)= \begin{cases}0 & \text { if } x<a \\ f(z, x) & \text { if } x \geq a\end{cases}
$$


Set $\hat{F}(z, x)=\int_{0}^{x} \hat{f}(z, s) d s$ and let $\hat{\varphi}: W_{0}^{1, p}(Z) \rightarrow \mathbb{R}$ be the functional defined by

$$
\hat{\varphi}(x)=\frac{1}{p}\|D x\|_{p}^{p}-\int_{Z} \hat{F}(z, x(z)) d z \quad \text { for all } x \in W_{0}^{1, p}(Z) .
$$

Clearly $\hat{\varphi} \in C^{1}\left(W_{0}^{1, p}(Z)\right)$.

Proposition 3.4. If hypotheses $H_{1}$ hold then problem (1-1) has a solution

$$
x_{0} \in \operatorname{int} C_{+}
$$

which is a local minimizer of the functional $\hat{\varphi}$.

Proof. Exploiting the compact embedding of $W_{0}^{1, p}(Z)$ into $L^{r}(Z)$, we can easily verify that the functional $\varphi_{+}$is sequentially weakly lower semicontinuous. Since $\varphi_{+}$is coercive (see Proposition 3.3), we can apply the Weierstrass theorem and obtain $x_{0} \in W_{0}^{1, p}(Z)$ such that

$$
\varphi_{+}\left(x_{0}\right)=m_{+}=\inf \varphi_{+} .
$$

We claim that $m_{+}<0$. Indeed, since $u_{1} \in \operatorname{int} C_{+}$, we can find $t>0$ small such that

(3-11) $0 \leq t u_{1}(z) \leq \delta_{0} \quad$ for all $z \in \bar{Z}$

$$
\begin{aligned}
& \Rightarrow F_{+}\left(z, t u_{1}(z)\right) \geq \frac{\xi_{0}}{p} t^{p} u_{1}(z)^{p} \quad \text { a.e. on } Z\left(\text { see } H_{1}(\mathrm{vi})\right) \\
& \left.\Rightarrow \varphi_{+}\left(t u_{1}\right) \leq \frac{t^{p}}{p}\left(\lambda_{1}-\xi_{0}\right)<0 \quad \text { (since }\left\|D u_{1}\right\|_{p}^{p}=\lambda_{1}\left\|u_{1}\right\|_{p}^{p}\right) \Rightarrow m_{+}<0 .
\end{aligned}
$$

Hence

$$
\varphi_{+}\left(x_{0}\right)=m_{+}<0=\varphi_{+}(0) \quad(\text { see }(3-10) \text { and }(3-11)) \Rightarrow x_{0} \neq 0 .
$$

From (3-10) we have

$$
\varphi_{+}^{\prime}\left(x_{0}\right)=0 \Rightarrow A\left(x_{0}\right)=N_{+}\left(x_{0}\right)
$$

where $N_{+}(u)(\cdot)=f_{+}(\cdot, u(\cdot))$ for all $u \in W_{0}^{1, p}(Z)$.

On (3-12) we act with $-x_{0}^{-} \in W_{0}^{1, p}(Z)$ and obtain

$\left\|D x_{0}^{-}\right\|_{p}^{p}=0 \quad$ (recall $f_{+}(z, x)=0$ for a.a. $z \in Z$, all $\left.x \leq 0\right) \Rightarrow x_{0} \geq 0, x_{0} \neq 0$.

From (3-9) we have

$$
\left.-\Delta_{p} x_{0}(z)=f_{+}\left(z, x_{0}(z)\right) \geq 0 \quad \text { a.e. on } Z \text { (see } H_{1}(\mathrm{iv})\right) \text {. }
$$

Nonlinear regularity theory (see [Lieberman 1988]) implies that $x_{0} \in C_{+} \backslash\{0\}$. Moreover, from (3-13) we have

$$
\Delta_{p} x_{0}(z) \leq 0 \quad \text { a.e. on } Z \text {. }
$$


Invoking the nonlinear strong maximum principle of Vázquez, we conclude that

$$
x_{0} \in \operatorname{int} C_{+} .
$$

Note that $\left.\varphi_{+}\right|_{C_{+}}=\left.\hat{\varphi}\right|_{C_{+}}$. So it follows that $x_{0}$ is a local $C_{0}^{1}(\bar{Z})$-minimizer of $\hat{\varphi}$. Then, by [García Azorero et al. 2000, Theorem 1], $x_{0}$ is also a local $W_{0}^{1, p}(Z)$ minimizer of $\hat{\varphi}$.

Next we produce a negative solution. For this purpose, we introduce the following truncation of the nonlinearity $f(z, \cdot)$ :

$$
\hat{f}_{-}(z, x)= \begin{cases}0 & \text { if } x \leq a \\ f(z, x) & \text { if } a \leq x \leq 0, \\ 0 & \text { if } x \geq 0\end{cases}
$$

We set $\hat{F}_{-}(z, x)=\int_{0}^{x} \hat{f}_{-}(z, s) d s$ and then define the functional

$$
\begin{gathered}
\hat{\varphi}_{-}: W_{0}^{1, p}(Z) \rightarrow \mathbb{R} \\
\hat{\varphi}_{-}(x)=\frac{1}{p}\|D x\|_{p}^{p}-\int_{Z} \hat{F}_{-}(z, x(z)) d z \quad \text { for all } x \in W_{0}^{1, p}(Z) .
\end{gathered}
$$

Again we have $\hat{\varphi}_{-} \in C^{1}\left(W_{0}^{1, p}(Z)\right)$.

Proposition 3.5. If hypotheses $H_{1}$ hold then problem (1-1) has a solution

$$
v_{0} \in-\operatorname{int} C_{+}
$$

which is a local minimizer of the functional $\hat{\varphi}_{-}$.

Proof. Clearly $\hat{\varphi}_{-}$is coercive and it is also sequentially weakly lower semicontinuous. Therefore, we can find $v_{0} \in W_{0}^{1, p}(Z)$ such that

$$
\hat{\varphi}_{-}\left(v_{0}\right)=\hat{m}_{-}=\inf \hat{\varphi}_{-}
$$

by the Weierstrass theorem.

As we did for $\varphi_{+}$(see the proof of Proposition 3.4), by choosing $t>0$ small such that

$$
\max \left\{-\delta_{0}, a\right\} \leq-t u_{1}(z) \leq 0
$$

for all $z \in \bar{Z}$, we can show using hypothesis $H_{1}$ (vi) that

$$
\hat{\varphi}_{-}\left(v_{0}\right)=\hat{m}_{-}<0=\hat{\varphi}_{-}(0) \Rightarrow v_{0} \neq 0 .
$$

From (3-14), we have

$$
A\left(v_{0}\right)=\hat{N}_{-}\left(v_{0}\right)
$$

where $\hat{N}_{-}(u)(\cdot)=\hat{f}_{-}(\cdot, u(\cdot))$ for all $u \in W_{0}^{1, p}(Z)$. 
On (3-15) we act with $v_{0}^{+} \in W_{0}^{1, p}(Z)$ and obtain

$\left\|D v_{0}^{+}\right\|_{p}^{p}=0 \quad$ (since $\hat{f}_{-}(z, x)=0$ for a.a. $z \in Z$, all $\left.x \geq 0\right) \Rightarrow v_{0} \leq 0, v_{0} \neq 0$.

Also from (3-15) we have

$$
\begin{array}{r}
\Delta_{p}\left(-v_{0}(z)\right)=-\Delta_{p} v_{0}(z)=\hat{f}_{-}\left(z, v_{0}(z)\right) \leq 0 \quad \text { a.e. on } Z\left(\text { see } H_{1}(\mathrm{v})\right) \\
\Rightarrow v_{0} \in-\operatorname{int} C_{+} \quad \text { (see [Vázquez 1984]). }
\end{array}
$$

If we act with $\left(a-v_{0}\right)^{+} \in W_{0}^{1, p}(Z)$ on (3-15), we obtain

$$
\begin{aligned}
\left\langle A\left(v_{0}\right),\left(a-v_{0}\right)^{+}\right\rangle & =\int_{\left\{a>v_{0}\right\}} \hat{f}_{-}\left(z, v_{0}\right)\left(a-v_{0}\right) d z=0 \\
& \Rightarrow\left\|D\left(a-v_{0}\right)^{+}\right\|_{p}^{p}=0 \Rightarrow a \leq v_{0} .
\end{aligned}
$$

By virtue of hypothesis $H_{1}(\mathrm{v})$, we have

$$
\begin{aligned}
-\Delta_{p} v_{0}(z) & +\xi\left|v_{0}(z)\right|^{p-2} v_{0}(z) \\
& =f\left(z, v_{0}(z)\right)+\xi\left|v_{0}(z)\right|^{p-2} v_{0}(z) \geq \xi|a|^{p-2} a \quad \text { a.e. on } Z(\text { see (3-16)) } \\
& \Rightarrow \Delta_{p} v_{0}(z)+\xi\left(|a|^{p-2} a-\left|v_{0}(z)\right|^{p-2} v_{0}(z)\right) \leq 0 \quad \text { a.e. on } Z .
\end{aligned}
$$

Involving the tangency principle of Serrin [1970] (see also [Pucci and Serrin 2007, p. 35]), we obtain

$$
v_{0}(z)>a \quad \text { for all } z \in \bar{Z} .
$$

Then it follows from the definition of $\hat{\varphi}_{-}$that we can find $\varrho>0$ such that if

$$
\bar{B}_{\varrho}^{C_{0}^{1}(\bar{Z})}\left(v_{0}\right)=\left\{u \in C_{0}^{1}(\bar{Z}):\left\|u-v_{0}\right\|_{C_{0}^{1}(\bar{Z})} \leq \varrho\right\},
$$

then

$$
\left.\hat{\varphi}_{-}\right|_{\bar{B}_{\varrho}} C_{0}^{1}(\bar{Z})=\left.\hat{\varphi}\right|_{\bar{B}_{\varrho} C_{0}^{1}(\bar{Z})} .
$$

Hence $v_{0} \in-\operatorname{int} C_{+}$is a local $C_{0}^{1}(\bar{Z})$-minimizer of $\hat{\varphi}$. Once again, Theorem 1 of [García Azorero et al. 2000], implies that

$$
v_{0} \in-\operatorname{int} C_{+}
$$

is a local $W_{0}^{1, p}(Z)$-minimizer of $\hat{\varphi}$.

Now using minimax techniques from critical point theory, we can produce a third nontrivial smooth solution of (1-1) and have the full multiplicity result (three solutions theorem) for problem (1-1).

Theorem 3.6. If hypotheses $H_{1}$ hold then problem (1-1) has at least three nontrivial smooth solutions

$$
x_{0} \in \operatorname{int} C_{+}, \quad v_{0} \in-\operatorname{int} C_{+} \quad \text { and } \quad y_{0} \in C_{0}^{1}(\bar{Z}) .
$$


Proof. From Propositions 3.4 and 3.5 we already have two constant sign solutions

$$
x_{0} \in \operatorname{int} C_{+}, \quad v_{0} \in-\operatorname{int} C_{+} .
$$

Without any loss of generality, we may assume that

$$
\hat{\varphi}\left(v_{0}\right) \leq \hat{\varphi}\left(x_{0}\right) .
$$

Also arguing as in [Motreanu et al. 2007, proof of Proposition 6], we can find $\rho>0$ small such that

$$
\left\|v_{0}-x_{0}\right\|>\rho \quad \text { and } \quad \hat{\varphi}\left(x_{0}\right)<\inf \left\{\hat{\varphi}(x):\left\|x-x_{0}\right\|=\rho\right\}=\eta_{\rho} .
$$

Claim 3.7. $\hat{\varphi}$ satisfies the PS-condition.

Proof of Claim 3.7. Let $\left\{x_{n}\right\}_{n \geq 1} \subseteq W_{0}^{1, p}(Z)$ be a sequence such that

$$
\begin{aligned}
& \left|\hat{\varphi}\left(x_{n}\right)\right| \leq M_{4} \quad \text { for some } M_{4}>0, \text { all } n \geq 1 \text { and } \\
& \hat{\varphi}^{\prime}\left(x_{n}\right) \rightarrow 0 \quad \text { in } W^{-1, p^{\prime}}(Z)\left(\frac{1}{p}+\frac{1}{p^{\prime}}=1\right) \text { as } n \rightarrow \infty .
\end{aligned}
$$

From (3-20), we have

(3-21) $\left|\left\langle\hat{\varphi}^{\prime}\left(x_{n}\right), u\right\rangle\right| \leq \varepsilon_{n}\|u\| \quad$ for all $u \in W_{0}^{1, p}(Z)$ with $\varepsilon_{n} \downarrow 0$

$$
\Rightarrow\left|\left\langle A\left(x_{n}\right), u\right\rangle-\int_{Z} \hat{f}\left(z, x_{n}\right) u d z\right| \leq \varepsilon_{n}\|u\| \text { for all } u \in W_{0}^{1, p}(Z) \text { with } \varepsilon_{n} \downarrow 0 \text {. }
$$

In (3-21) we choose $u=-x_{n}^{-} \in W_{0}^{1, p}(Z)$. Recalling the definition of $\hat{f}(z, x)$, we have

$$
\begin{aligned}
\left\|D x_{n}^{-}\right\|_{p}^{p}-\int_{Z} \hat{f}_{-}(z, & \left.-x_{n}^{-}(z)\right)\left(-x_{n}^{-}(z)\right) d z \leq \varepsilon_{n}\left\|x_{n}^{-}\right\| \\
& \Rightarrow\left\|D x_{n}^{-}\right\|_{p}^{p} \leq c_{3}\left\|x_{n}^{-}\right\| \quad \text { for some } c_{3}>0, \text { all } n \geq 1 \\
& \Rightarrow\left\{x_{n}^{-}\right\}_{n \geq 1} \subseteq W_{0}^{1, p}(Z) \text { is bounded. }
\end{aligned}
$$

From (3-19) and (3-22) we have

$$
\begin{aligned}
\frac{1}{p}\left\|D x_{n}^{+}\right\|_{p}^{p}-\int_{Z} F_{+}\left(z, x_{n}^{+}(z)\right) d z \leq M_{5} & \text { for some } M_{5}>0, \text { all } n \geq 1 \\
& \Rightarrow \varphi_{+}\left(x_{n}^{+}\right) \leq M_{5} \text { for all } n \geq 1 .
\end{aligned}
$$

But from Proposition 3.3, we know that $\varphi_{+}$is coercive. Hence, from (3-23) it follows that

$$
\left\{x_{n}^{+}\right\}_{n \geq 1} \subseteq W_{0}^{1, p}(Z) \text { is bounded }
$$


From (3-22) and (3-24) we infer that $\left\{x_{n}\right\}_{n \geq 1} \subseteq W_{0}^{1, p}(Z)$ is bounded. So we may assume that

$$
x_{n} \stackrel{w}{\rightarrow} x \text { in } W_{0}^{1, p}(Z) \quad \text { and } \quad x_{n} \rightarrow x \text { in } L^{r}(Z) .
$$

From (3-21) we have

$$
\left|\left\langle A\left(x_{n}\right), x_{n}-x\right\rangle-\int_{Z} \hat{f}\left(z, x_{n}\right)\left(x_{n}-x\right) d z\right| \leq \varepsilon_{n}\left\|x_{n}-x\right\| .
$$

Note that

$$
\int_{Z} \hat{f}\left(z, x_{n}\right)\left(x_{n}-x\right) d z \rightarrow 0
$$

as $n \rightarrow \infty$. So from (3-25) we have

$$
\lim _{n \rightarrow \infty}\left\langle A\left(x_{n}\right), x_{n}-x\right\rangle=0 \Rightarrow x_{n} \rightarrow x \text { in } W_{0}^{1, p}(Z) \text { (see Proposition 2.4). }
$$

Claim 3.7 follows.

Then (3-17), (3-18) and Claim 3.7 permit the use of Theorem 2.1 (the Mountain Pass Theorem). Therefore, we obtain $y_{0} \in W_{0}^{1, p}(Z)$ such that

$$
\begin{aligned}
\hat{\varphi}^{\prime}\left(y_{0}\right) & =0, \\
\hat{c}=\hat{\varphi}\left(y_{0}\right) & =\inf _{\gamma \in \Gamma} \max _{0 \leq t \leq 1} \varphi(\gamma(t)) \geq \eta_{\rho}>\hat{\varphi}\left(x_{0}\right) \geq \hat{\varphi}\left(v_{0}\right)
\end{aligned}
$$

where $\Gamma=\left\{\gamma \in C\left([0,1], W_{0}^{1, p}(Z)\right): \gamma(0)=v_{0}, \gamma(1)=x_{0}\right\}$.

From (3-27) it is clear that $y_{0} \neq x_{0}$ and $y_{0} \neq v_{0}$. We need to show that $y_{0}$ is nontrivial (that is, $y_{0} \neq 0$ ). According to the minimax expression in (3-27), it suffices to produce $\gamma_{*} \in \Gamma$ such that $\left.\hat{\varphi}\right|_{\gamma_{*}}<0$. Then

$$
\hat{c}=\hat{\varphi}\left(y_{0}\right)<0=\hat{\varphi}(0)
$$

and so $y_{0} \neq 0$.

So our goal is to produce such a path $\gamma_{*} \in \Gamma$. Let

$$
S=W_{0}^{1, p}(Z) \cap \partial B_{1}^{L^{p}}
$$

be endowed with the relative $W_{0}^{1, p}(Z)$-topology and let

$$
S_{c}=S \cap C_{0}^{1}(\bar{Z})
$$

be endowed with the relative $C_{0}^{1}(\bar{Z})$-topology. Also let

$$
\begin{aligned}
\hat{\Gamma} & =\left\{\hat{\gamma} \in(C[-1,1], S): \gamma(-1)=-u_{1}, \hat{\gamma}(1)=u_{1}\right\}, \\
\hat{\Gamma}_{c} & =\left\{\hat{\gamma} \in\left(C[-1,1], S_{c}\right): \gamma(-1)=-u_{1}, \hat{\gamma}(1)=u_{1}\right\} .
\end{aligned}
$$


The density of $S_{c}$ in $S$ for the $W_{0}^{1, p}(Z)$-topology implies the density of $\hat{\Gamma}_{c}$ in $\hat{\Gamma}$ for the $C([-1,1], S)$-topology. From (2-5) we see that given any $\delta>0$ we can find $\hat{\gamma}_{0}=\hat{\gamma}_{0}(\delta) \in \hat{\Gamma}_{c}$ such that

$$
\max _{-1 \leq t \leq 1}\left\|D \hat{\gamma}_{0}(t)\right\|_{p}^{p} \leq \lambda_{2}+\delta .
$$

Since $\hat{\gamma}_{0} \in \hat{\Gamma}_{c}$, we can find $\varepsilon>0$ small such that

$$
\varepsilon\left|\hat{\gamma}_{0}(t)(z)\right| \leq \min \left\{\delta_{0},-a\right\} \quad \text { for all } t \in T \text { and all } z \in \bar{Z} .
$$

Then for all $t \in[0,1]$, we have

$$
\begin{aligned}
\hat{\varphi}\left(\varepsilon \hat{\gamma}_{0}(t)\right) & =\frac{\varepsilon^{p}}{p}\left\|D \hat{\gamma}_{0}(t)\right\|_{p}^{p}-\int_{Z} \hat{F}\left(z, \varepsilon \hat{\gamma}_{0}(t)(z)\right) d z \\
& \leq \frac{\varepsilon^{p}}{p}\left(\lambda_{2}+\delta\right)-\frac{\varepsilon^{p}}{p} \xi_{0}=\frac{\varepsilon^{p}}{p}\left(\lambda_{2}+\delta-\xi_{0}\right)
\end{aligned}
$$

(see (3-28), hypothesis $H_{1}$ (vi) and recall that $\left\|\hat{\gamma}_{0}(t)\right\|_{p}=1$ ).

We choose

$$
\delta<\xi_{0}-\lambda_{2}
$$

(hypothesis $H_{1}(\mathrm{vi})$ ). Then from (3-29) it follows that for $\hat{\gamma}_{0}^{\varepsilon}=\varepsilon \hat{\gamma}_{0}$, we have

$$
\left.\hat{\varphi}\right|_{\hat{\gamma}_{0}^{\varepsilon}}<0
$$

Next we will produce a continuous path from $\varepsilon u_{1}$ to $x_{0}$ along which $\hat{\varphi}$ is negative. Suppose that $\left\{0, x_{0}\right\}$ are the only critical points of the functional $\varphi_{+}$. Otherwise, we have one more critical point of $\varphi_{+}$, which as before we can check that it is in int $C_{+}$. Hence it is also a critical point of $\hat{\varphi}$ and thus it is a solution of (1-1). Therefore we have three constant sign solutions and we are done.

We set

$$
a=m_{+}<0=\varphi_{+}(0)=b .
$$

From Proposition 3.3 we know that $\varphi_{+}$is coercive. Therefore, $\varphi_{+}$satisfies the PS-condition (it can be verified as in Claim 3.7 in the proof of Theorem 3.6). Apply Theorem 2.2 (the Second Deformation Theorem) to obtain a continuous deformation

$$
h:[0,1] \times\left(\varphi_{+}^{b} \backslash K_{b}^{+}\right) \rightarrow \varphi_{+}^{b}
$$

where $K_{\theta}^{+}=\left\{x \in W_{0}^{1, p}(Z): \varphi_{+}^{\prime}(x)=0, \varphi_{+}(x)=\theta\right\}$ for every $\theta \in \mathbb{R}$, such that

$$
\left.h(t, \cdot)\right|_{K_{a}^{+}}=\left.\mathrm{id}\right|_{K_{a}^{+}}
$$


for all $t \in[0,1]$ and

$$
\begin{aligned}
h\left(1, \varphi_{+}^{b} \backslash K_{b}^{+}\right) & \subseteq \varphi_{+}^{a} \\
\varphi_{+}(h(t, x)) & \leq \varphi_{+}(h(s, x)) \quad \text { for all } 0 \leq s \leq t \leq 1, \text { all } x \in \varphi_{+}^{b} \backslash K_{b}^{+} .
\end{aligned}
$$

Let $\gamma_{+}(t)=h\left(t, \varepsilon u_{1}\right)$. Evidently $\gamma_{+} \in C\left([0,1], W_{0}^{1, p}(Z)\right)$. Also

$$
\begin{aligned}
\gamma_{+}(0) & =h\left(0, \varepsilon u_{1}\right)=\varepsilon u_{1} \quad(\text { since } h \text { is a deformation }), \\
\gamma_{+}(1) & =h\left(1, \varepsilon u_{1}\right)=x_{0} \quad\left(\text { see }(3-31) \text { and recall that } \varphi_{+}^{a}=\left\{x_{0}\right\}\right), \\
\varphi_{+}\left(\gamma_{+}(t)\right) & =\varphi_{+}\left(h\left(t, \varepsilon u_{1}\right)\right) \leq \varphi_{+}\left(h\left(0, \varepsilon u_{1}\right)\right) \\
& =\varphi_{+}\left(\varepsilon u_{1}\right)<0 \quad\left(\text { see }(3-32),(3-30) \text { and }\left.\hat{\varphi}\right|_{C_{+}}=\left.\varphi_{+}\right|_{C_{+}}\right) .
\end{aligned}
$$

Therefore, from the above we have that $\gamma_{+}$is a continuous path from $\varepsilon u_{1}$ to $x_{0}$ and

$$
\left.\varphi_{+}\right|_{\gamma_{+}}<0
$$

Recall that $f(z, x) \geq 0$ for a.a. $z \in Z$, all $x \in \mathbb{R}_{+}$and $f(z, x) \leq 0$ for a.a. $z \in Z$, all $x \in[a, 0]$. So it follows that $\hat{\varphi} \leq \varphi_{+}$and hence

$$
\left.\hat{\varphi}\right|_{\gamma_{+}}<0 \text {. }
$$

Finally, we produce a continuous path from $-\varepsilon u_{1}$ to $v_{0}$ along which $\hat{\varphi}$ is negative. Again, we may assume that $\left\{0, v_{0}\right\}$ are the only critical points of $\hat{\varphi}_{-}$. Otherwise, as before, we have a third nontrivial constant sign solution (in $-\operatorname{int} C_{+}$) of (1-1) and so we are done. We set

$$
a=\hat{m}_{-}<0=\hat{\varphi}_{-}(0)=b .
$$

The functional $\hat{\varphi}_{-}$is coercive; hence, it satisfies the PS-condition. We apply Theorem 2.2 (the Second Deformation Theorem) and obtain a continuous deformation

$$
\hat{h}:[0,1] \times\left(\hat{\varphi}_{-}^{b} \backslash \hat{K}_{b}^{-}\right) \rightarrow \hat{\varphi}_{-}^{b}
$$

with the similar properties as before, where

$$
\hat{K}_{\theta}^{-}=\left\{x \in W_{0}^{1, p}(Z): \hat{\varphi}_{-}^{\prime}(x)=0, \hat{\varphi}_{-}(x)=\theta\right\}
$$

for every $\theta \in \mathbb{R}$. We set $\gamma_{-}(t)=\hat{h}\left(t,-\varepsilon u_{1}\right)$ and as we did for $\gamma_{+}$, we check that $\gamma_{-}$is a continuous path from $-\varepsilon u_{1}$ to $v_{0}$ such that

$$
\left.\hat{\varphi}_{-}\right|_{\gamma_{-}}<\left.0 \Rightarrow \hat{\varphi}\right|_{\gamma_{-}}<0 \quad\left(\text { since } \hat{\varphi} \leq \hat{\varphi}_{-} ; \text {see hypothesis } H_{1}(\mathrm{v})\right) .
$$

We concatenate $\gamma_{-}, \hat{\gamma}_{0}^{\varepsilon}$ and $\gamma_{+}$and obtain $\gamma_{*} \in \Gamma$ such that

$$
\left.\hat{\varphi}\right|_{\gamma_{*}}<0 \quad \text { (see (3-30), (3-33), (3-34)) } \Rightarrow y_{0} \neq 0 \text {. }
$$


From (3-26), we have

$$
\begin{aligned}
A\left(y_{0}\right)=\hat{N}\left(y_{0}\right) & \Rightarrow-\Delta_{p} y_{0}(z)=\hat{f}\left(z, y_{0}(z)\right) \quad \text { a.e. on } Z \\
& \Rightarrow y_{0} \in C_{0}^{1}(\bar{Z}) \backslash\{0\} \quad \text { (nonlinear regularity theory). }
\end{aligned}
$$

As in the proof of Proposition 3.5, acting with $\left(a-y_{0}\right)^{+} \in W_{0}^{1, p}(Z)$, we obtain $a \leq y_{0}$ and hence $y_{0}$ is a nontrivial smooth solution of (1-1).

\section{The semilinear problem}

In this section, we focus on the semilinear problem (that is, $p=2$ ). So, the problem under consideration is

$$
\begin{aligned}
-\Delta x(z) & =f(z, x(z))) \quad \text { a.e. on } Z, \\
\left.x\right|_{\partial Z} & =0 .
\end{aligned}
$$

By strengthening the regularity on $f(z, \cdot)$ and using Morse theory, we can show that the problem has four nontrivial smooth solutions.

Now the nonlinearity hypotheses on $f(z, x)$ are the following:

$\underline{H_{2}}: f: Z \times \mathbb{R} \rightarrow \mathbb{R}$ is a function such that $f(z, 0)=0$ a.e. on $Z$.

(i) For all $x \in \mathbb{R}, z \rightarrow f(z, x)$ is measurable.

(ii) For almost all $z \in Z, x \rightarrow f(z, x)$ is $C^{1}$.

(iii) For almost all $z \in Z$ and all $x \in \mathbb{R}$,

$$
\left|f_{x}^{\prime}(z, x)\right| \leq a(z)+c|x|^{r-2}
$$

with $a \in L^{\infty}(Z)_{+}, c>0$ and $2<r<2^{*}$.

(iv) For almost all $z \in Z$ and all $x \geq 0, f(z, x) \geq 0$ and

$$
\lim _{x \rightarrow+\infty} \frac{2 F(z, x)}{x^{2}}=\lambda_{1} \text { and } \lim _{x \rightarrow+\infty}\left(2 F(z, x)-\lambda_{1} x^{2}\right)=-\infty,
$$

both uniformly for almost all $z \in Z$.

(v) There exists $a<0$ such that $f(z, a)=0$ a.e. on $Z$ and $f(z, x) \leq 0$ for a.a. $z \in Z$ and all $x \in[a, 0]$.

(vi) There exist $\delta_{0}>0$ and an integer $m \geq 2$ such that

$$
\lambda_{m} \leq \frac{f(z, x)}{x} \leq \lambda_{m+1}
$$

for a.a. $z \in Z$, all $|x| \leq \delta_{0}$ and if $m=2$, then in addition

$$
\lambda_{2}<f_{x}^{\prime}(z, 0)=\lim _{x \rightarrow 0} \frac{f(z, x)}{x}
$$

uniformly for almost all $z \in Z$. 
Remark 4.1. Evidently hypotheses $H_{2}$ (ii)-(iii) imply that we can find $\xi_{0}>0$ large such that for almost all $z \in Z, x \rightarrow \xi_{0} x+f(z, x)$ is nondecreasing on $[a, 0]$.

Example 4.2. The following function $f(x)$ satisfies hypotheses $\mathrm{H}_{2}$. Again for the sake of simplicity we drop the $z$-dependence:

$$
f(x)= \begin{cases}x^{2}+x & \text { if } x \leq 0, \\ c\left(x-x^{2}\right) & \text { if } x \in[0,1], \\ \lambda_{1} x-\left(\lambda_{1}+c\right) \ln x-\lambda_{1} & \text { if } x \geq 1,\end{cases}
$$

with $\lambda_{m} \leq c \leq \lambda_{m+1}, m \geq 3$.

Theorem 4.3. If hypothesis $\mathrm{H}_{2}$ hold then problem (4-1) has at least four nontrivial solutions

$$
x_{0} \in \operatorname{int} C_{+}, \quad v_{0} \in-\operatorname{int} C_{+}, \quad \text { and } \quad y_{0}, u_{0} \in C_{0}^{1}(\bar{Z}) .
$$

Proof. From Theorem 3.6, we already have three nontrivial smooth solutions

$$
x_{0} \in \operatorname{int} C_{+}, \quad v_{0} \in-\operatorname{int} C_{+}, \quad y_{0} \in C_{0}^{1}(\bar{Z}) .
$$

From Propositions 3.4 and 3.5 we know that $x_{0}, v_{0}$ are both local minimizers of $\hat{\varphi}$. Therefore

$$
C_{k}\left(\hat{\varphi}, x_{0}\right)=C_{k}\left(\hat{\varphi}, v_{0}\right)=\delta_{k, 0} \mathbb{Z} \quad \text { for all } k \geq 0
$$

(see [Chang 1993, p. 33] and [Mawhin and Willem 1989, p. 175]).

Hypothesis $H_{2}$ (vi) and [Li et al. 2001, Proposition 1.1] imply that

$$
C_{k}(\hat{\varphi}, 0)=\delta_{k, d_{m}} \mathbb{Z}
$$

for all $k \geq 0$, where

$$
d_{m}=\operatorname{dim} \bigoplus_{i=1}^{m} E\left(\lambda_{i}\right)
$$

( $E\left(\lambda_{i}\right)$ is the eigenspace corresponding to the eigenvalue $\lambda_{i}$ ). From the proof of Theorem 3.6, we know that $y_{0} \in C_{0}^{1}(\bar{Z}) \backslash\{0\}$ was obtained via the use of the mountain pass theorem (see Theorem 2.1). Moreover, as before, using the maximum principle of Vázquez, we obtain $a<y_{0}(z)$ for all $z \in \bar{Z}$. Note that $\hat{\varphi} \in C^{2-0}\left(H_{0}^{1}(Z)\right)$. The fact that $\hat{\varphi}$ is not necessarily $C^{2}$ does not allow the direct use of well-known results from Morse theory. We overcome this inconvenience by approximating $\hat{\varphi}$ with a $C^{2}$-functional $\varphi_{0}$, keeping the essential properties intact. Note that the nonlinearity $f(z, \cdot)$ need not be $C^{1}$ only at $x=a$. So we approximate $\hat{f}(z, x)$ by a Caratheodory function $f_{0}(z, x)$ which is $C^{1}$ in the $x$-variable, differs from $\hat{f}(z, \cdot)$ only near $a$ and for a given $\varepsilon>0$, we have

$$
\int_{Z-r \leq x \leq r} \sup _{-}\left|\hat{f}(z, x)-f_{0}(z, x)\right| d z<\varepsilon \quad \text { for all } r>0 .
$$


We let $\varphi_{0}$ be the $C^{2}$-functional corresponding to $f_{0}(z, x)$.

Then, exploiting the continuity of the Morse critical groups in the $C^{1}$-norm (see [Chang 2005, p. 337]), we have

$$
C_{k}\left(\left.\hat{\varphi}\right|_{C_{0}^{1}(\bar{Z})}, y_{0}\right)=C_{k}\left(\left.\varphi_{0}\right|_{C_{0}^{1}(\bar{Z})}, y_{0}\right) \quad \text { for all } k \geq 0 .
$$

But from [Liu and Wu 2002], we know that, for all $k \geq 0$,

$$
C_{k}\left(\hat{\varphi}, y_{0}\right)=C_{k}\left(\left.\hat{\varphi}\right|_{C_{0}^{1}(\bar{Z})}, y_{0}\right) \quad \text { and } \quad C_{k}\left(\hat{\varphi}_{0}, y_{0}\right)=C_{k}\left(\left.\varphi_{0}\right|_{C_{0}^{1}(\bar{Z})}, y_{0}\right) .
$$

From (4-4) and (4-5), we infer that

$$
C_{k}\left(\hat{\varphi}, y_{0}\right)=C_{k}\left(\varphi_{0}, y_{0}\right) \quad \text { for all } k \geq 0 .
$$

But $\varphi_{0} \in C^{2}\left(H_{0}^{1}(Z)\right)$ and $\varphi_{0}^{\prime \prime}\left(y_{0}\right)$ is a Fredholm operator. Suppose that the Morse index of $\varphi_{0}$ at $y_{0}$ is zero. Then

$$
\|D u\|_{2}^{2} \geq \int_{Z} m u^{2} d z
$$

for all $u \in H_{0}^{1}(Z)$, where $m(z)=f_{0}^{\prime}\left(z, y_{0}(z)\right)$ and $m \in L^{\infty}(Z)$. Let $u \in \operatorname{ker} \varphi_{0}^{\prime \prime}\left(y_{0}\right)$. Then

$$
-\Delta u(z)=m(z) u(z) \quad \text { a.e. on } Z,\left.\quad u\right|_{\partial Z}=0 .
$$

If $m^{+}=0$ then clearly (4-8) has only the trivial solution. If $m^{+} \neq 0$ then from the variational characterization of the principal eigenvalue $\hat{\lambda}_{1}(m)$ of $\left(-\Delta, H_{0}^{1}(Z), m\right)$, we have

$$
\lambda_{1}(m) \geq 1 \quad(\text { see }(4-7)) \Rightarrow \operatorname{dim} \operatorname{ker} \varphi^{\prime \prime}\left(y_{0}\right) \leq 1 \quad(\text { see }(4-8)) .
$$

So we apply [Mawhin and Willem 1989, Corollary 8.5, p. 195] and obtain:

$C_{k}\left(\varphi_{0}, y_{0}\right)=\delta_{k, 1} \mathbb{Z} \quad$ for all $k \geq 0 \Rightarrow C_{k}\left(\hat{\varphi}, y_{0}\right)=\delta_{k, 1} \mathbb{Z} \quad$ for all $k \geq 0$ (see (4-6)).

Claim 4.4. $\hat{\varphi}$ is coercive.

Proof. We argue indirectly. So suppose that Claim 4.4 is not true. Then we can find $\left\{x_{n}\right\}_{n \geq 1} \subseteq H_{0}^{1}(Z)$ and $M_{6}>0$ such that

(4-10) $\quad\left\|x_{n}\right\| \rightarrow \infty \quad$ as $n \rightarrow \infty \quad$ and $\quad \hat{\varphi}\left(x_{n}\right) \leq M_{6} \quad$ for all $n \geq 1$.

Then, recalling the definition of $\hat{f}(z, x)$, we have

$$
\varphi_{+}\left(x_{n}\right)=\frac{1}{2}\left\|D x_{n}\right\|_{2}^{2}-\int_{Z} F_{+}\left(z, x_{n}\right) d z \leq M_{7} \quad \text { for some } M_{7}>0, \text { all } n \geq 1,
$$

which contradicts Proposition 3.3 (see (4-10)). 
Using Claim 4.4 and directly from the definition of the critical groups of $\hat{\varphi}$ at infinity, we have

$$
C_{k}(\hat{\varphi}, \infty)=\delta_{k, 0} \mathbb{Z} \quad \text { for all } k \geq 0 .
$$

Suppose that $\left\{0, x_{0}, v_{0}, y_{0}\right\}$ are all the critical points of $\hat{\varphi}$. Then from (2-1) with $t=-1$, we have

$2(-1)^{0}+(-1)^{d_{m}}+(-1)^{1}=(-1)^{0} \quad($ see $(4-2),(4-3),(4-9),(4-11)) \Rightarrow(-1)^{d_{m}}=0$,

a contradiction. So there is a fourth nontrivial critical point $u_{0} \in H_{0}^{1}(Z)$ of $\hat{\varphi}$. We can show that $u_{0} \geq a$; hence, it solves (4-1) and by regularity theory $u_{0} \in C_{0}^{1}(\bar{Z})$.

\section{Acknowledgement}

The authors wish to thank the referee for his/her constructive criticism and remarks.

\section{References}

[Bartsch and Li 1997] T. Bartsch and S. Li, "Critical point theory for asymptotically quadratic functionals and applications to problems with resonance”, Nonlinear Anal. 28:3 (1997), 419-441. MR 98k:58041 Zbl 0872.58018

[Carl and Perera 2002] S. Carl and K. Perera, "Sign-changing and multiple solutions for the $p$ Laplacian", Abstr. Appl. Anal. 7:12 (2002), 613-625. MR 2004a:35061 Zbl 1106.35308

[Chang 1993] K.-c. Chang, Infinite-dimensional Morse theory and multiple solution problems, Progress in Nonlinear Differential Equations and their Applications 6, Birkhäuser, Boston, 1993. MR 94e:58023 Zbl 0779.58005

[Chang 2005] K.-C. Chang, Methods in nonlinear analysis, Springer, Berlin, 2005. MR 2007b: $47169 \mathrm{Zbl} 1081.47001$

[Cuesta 2001] M. Cuesta, "Eigenvalue problems for the $p$-Laplacian with indefinite weights", Electron. J. Differential Equations 2001:33 (2001), 1-9. MR 2002b:35165 Zbl 0964.35110

[Cuesta et al. 1999] M. Cuesta, D. de Figueiredo, and J.-P. Gossez, "The beginning of the Fučik spectrum for the p-Laplacian”, J. Differential Equations 159:1 (1999), 212-238. MR 2001f:35308 Zbl 0947.35068

[García Azorero et al. 2000] J. P. García Azorero, I. Peral Alonso, and J. J. Manfredi, "Sobolev versus Hölder local minimizers and global multiplicity for some quasilinear elliptic equations", Commun. Contemp. Math. 2:3 (2000), 385-404. MR 2001k:35062

[Gasiński and Papageorgiou 2006] L. Gasiński and N. S. Papageorgiou, Nonlinear analysis, Series in Mathematical Analysis and Applications 9, Chapman \& Hall/CRC, Boca Raton, FL, 2006. MR 2006e:47001 Zbl 1086.47001

[Guo and Liu 2005] Y. Guo and J. Liu, "Solutions of $p$-sublinear $p$-Laplacian equation via Morse theory", J. London Math. Soc. (2) 72:3 (2005), 632-644. MR 2006j:35078 Zbl 02246563

[Lê 2006] A. Lê, "Eigenvalue problems for the p-Laplacian", Nonlinear Anal. 64:5 (2006), 10571099. MR 2007b:35246 Zbl 1128.35347

[Li et al. 2001] S. Li, K. Perera, and J. Su, "Computation of critical groups in elliptic boundaryvalue problems where the asymptotic limits may not exist", Proc. Roy. Soc. Edinburgh Sect. A 131:3 (2001), 721-732. MR 2002g:35082 Zbl 1114.35321 
[Lieberman 1988] G. M. Lieberman, "Boundary regularity for solutions of degenerate elliptic equations”, Nonlinear Anal. 12:11 (1988), 1203-1219. MR 90a:35098 Zbl 0675.35042

[Liu 2006] S. Liu, "Multiple solutions for coercive p-Laplacian equations", J. Math. Anal. Appl. 316:1 (2006), 229-236. MR 2006m:35103 Zbl 1148.35321

[Liu and Liu 2005] J. Liu and S. Liu, "The existence of multiple solutions to quasilinear elliptic equations”, Bull. London Math. Soc. 37:4 (2005), 592-600. MR 2006m:35102 Zbl 1122.35033

[Liu and $\mathrm{Wu}$ 2002] J. Liu and S. Wu, "Calculating critical groups of solutions for elliptic problem with jumping nonlinearity”, Nonlinear Anal. Ser. A 49:6 (2002), 779-797. MR 2003b:35080 Zbl 1012.35022

[Mawhin and Willem 1989] J. Mawhin and M. Willem, Critical point theory and Hamiltonian systems, Applied Mathematical Sciences 74, Springer, New York, 1989. MR 90e:58016 Zbl 0676. 58017

[Motreanu et al. 2007] D. Motreanu, V. V. Motreanu, and N. S. Papageorgiou, "A degree theoretic approach for multiple solutions of constant sign for nonlinear elliptic equations", Manuscripta Math. 124:4 (2007), 507-531. MR 2357796 Zbl 1148.35031

[Papageorgiou and Papageorgiou 2007] E. H. Papageorgiou and N. S. Papageorgiou, "A multiplicity theorem for problems with the p-Laplacian", J. Funct. Anal. 244:1 (2007), 63-77. MR 2008e:35074 Zbl 1147.35033

[Pucci and Serrin 2007] P. Pucci and J. Serrin, The maximum principle, Progress in Nonlinear Differential Equations and their Applications 73, Birkhäuser Verlag, Basel, 2007. MR 2008m:35001 Zbl 1134.35001

[Serrin 1970] J. Serrin, “On the strong maximum principle for quasilinear second order differential inequalities”, J. Functional Analysis 5 (1970), 184-193. MR 41 \#3966 Zbl 0188.41701

[Vázquez 1984] J. L. Vázquez, "A strong maximum principle for some quasilinear elliptic equations", Appl. Math. Optim. 12:3 (1984), 191-202. MR 86m:35018 Zbl 0561.35003

[Zhang et al. 2004] Z. Zhang, J. Chen, and S. Li, "Construction of pseudo-gradient vector field and sign-changing multiple solutions involving $p$-Laplacian”, J. Differential Equations 201:2 (2004), 287-303. MR 2006d:35047 Zbl 1079.35035

Received October 5, 2008. Revised November 27, 2008.

\title{
Evgenia H. Papageorgiou \\ DEPARTMENT OF MATHEMATICS \\ NATIONAL TECHNICAL UNIVERSITY \\ ZOGRAFOU CAMPUS \\ 15780 ATHENS \\ GREECE
}

epap@math.ntua.gr

\author{
Nicolaos S. Papageorgiou \\ DEPARTMENT OF MATHEMATICS \\ NATIONAL TECHNICAL UNIVERSITY \\ ZOGRAFOU CAMPUS \\ 15780 ATHENS \\ GREECE \\ npapg@math.ntua.gr
}

